\title{
Estilos y representaciones de apego en consumidores de drogas
}

\author{
Maa Teresa de Lucas Taracena; Francisco Montañés Rada \\ Psiquiatras. Fundación Hospital Alcorcón, Madrid. \\ Enviar correspondencia a: \\ Ma Teresa de Lucas Taracena. C/ Melilla, 29 B, $1^{\circ}$ A. 28005 Madrid. e-mail: pacoxmayte@yahoo.es
}

Recibido: 29 de noviembre de 2005. Aceptado: 13 de agosto de2006

\section{RESUMEN}

El apego inseguro se ha revelado factor de riesgo de diversos problemas de salud mental. Parece plausible que el apego inseguro, en general desarrollado en la infancia, es un factor de riesgo de trastornos por consumo de drogas (TCDs), y esto podría también afectar la alianza terapéutica, y secundariamente, la evolución del TCD. Hemos revisado la literatura sobre este tema, buscando relaciones entre patrones de apego y TCDs. Los estudios publicados sobre apego y TCDs. han producido resultados poco consistentes, en parte por el uso de diferentes medidas de evaluación. Los estudios con los autoinformes de Hazan y Shaver mostraron relación sobre todo con estilo de apego evasivo. Los estudios con la Entrevista de Apego del Adulto encontraron patrones de apego devaluadores o no resueltos. Finalmente, los estudios con el modelo de Bartholomew, en consumidores de alcohol u opiáceos, han encontrado estilos preocupados o temerosos. Discusión y conclusiones: Los estudios analizados son heterogéneos en cuanto a características de la muestra que a veces incluso no se especifican (edad, tipo de droga consumida, gravedad de la adicción) y al uso de instrumentos que clasifican de modo diferente las categorías de apego. No obstante, el apego seguro es similar en todos los instrumentos y no parece explicar los TCD. También hay pocos datos que apoyen una relación entre apego preocupado o ansioso y TCDs. Si bien algunos estudios apuntan a la existencia de patrones devaluadores de apego, la mayoría de trabajos encuentran apegos evasivos o temerosos.

Palabras clave: apego, factor de riesgo, abuso, dependencia, drogas, alcohol.

\begin{abstract}
Insecure attachment has been shown to be a risk factor for a variety of mental health problems. It seems plausible that insecure attachment, usually developing during childhood, is a risk factor for substance use disorders (SUDs), and it could also have an effect on the therapeutic alliance and, accordingly, on the SUD outcome. We have reviewed the literature on this topic, looking for attachment patterns linked to SUDs. Published studies on attachment and SUDs have produced inconsistent results, partly due to the use of different evaluation methods. Studies working with the Hazan and Shaver self-report, showed a link mainly with avoidant attachment style. Studies using the Adult Attachment Interview found dismissing or unresolved attachment patterns. Finally, studies of alcohol and opiate users with the Bartholomew model have found preoccupied or fearful styles. Discussion and conclusions: The reviewed studies are heterogeneous in respect of the sample characteristics that are, at times, not even specified (age, kind of drugs used, severity of the addiction), and the use of instruments that classify the attachment categories in a different way. Nevertheless, secure attachment is similar across all the measures and it does not seem to justify SUDs. In addition, there are few data supporting a relationship between preoccupied or anxious attachment, and SUDs. Although some studies point to the existence of dismissing attachment patterns, the majority of studies have found avoidant or fearful attachments.
\end{abstract}

Key words: attachment, risk factor, abuse, dependence, drug, abuse, alcohol.

\section{INTRODUCCIÓN}

$\mathbf{E}$ n general podemos definir el apego como la existencia de lazos emocionales duraderos que unen a una persona con otra significativa. Sería un sistema motivacional innato para mantener la proxi- midad entre el bebé y sus padres (primeras figuras de apego), y así asegurar la supervivencia del pequeño en situaciones de amenaza. Funcionaría de modo que el niño se sienta capaz de depender de ellos y que éstos a su vez sepan contener y proteger y dar seguridad al niño, especialmente en situaciones de mayor 
necesidad. Sobre la base de repetidas experiencias con sus figuras de apego, los niños desarrollan expectativas que se convierten en representaciones mentales o "modelos operantes", capaces de integrar experiencias pasadas y presentes, junto a esquemas cognitivos y emocionales relacionados con tales experiencias de modo que los niños pueden predecir e interpretar la conducta de sus figuras de apego. Estos modelos se integran a la estructura de la personalidad y proveen un prototipo para futuras relaciones sociales. Se construyen en los primeros años de vida y luego son susceptibles a reelaboraciones en función con las interacciones con figuras de apego de la persona, pero dentro de ciertos límites, puesto que las representaciones de las experiencias anteriores filtran las expectativas del individuo e influyen en su percepción de estas interacciones, haciendo que se establezca como rasgo persistente, lo que inicialmente nace como un simple estado.

A pesar de existir diversos estudios publicados sobre los estilos de apego presentes en sujetos con diferentes trastornos psiquiátricos o del comportamiento, son relativamente escasos los trabajos centrados directamente en los estilos de apego de los consumidores de alcohol y/o drogas. Muchos trabajos incluyen los trastornos por consumo de drogas (TCD) como un aspecto colateral, que no se investiga específicamente. Creemos, sin embargo, que el estilo de apego del sujeto puede tratarse de un aspecto esencial a la hora de establecer la alianza con el terapeuta, que es una relación interpersonal más y susceptible, por tanto, de mostrar características similares a las que el paciente establece con familia, amigos y otros miembros de su entorno, en cuanto a elementos tales como confianza, dependencia, comunicación de necesidades afectivas, etc. Y, a su vez, dicha relación terapéutica es la responsable en gran parte del éxito del tratamiento y el pronóstico del propio TCD.

Si bien somos conscientes de la inmensa variedad de tipos y formas de consumo, la existencia de consumos de sustancias de muy diferente acción farmacológica, y la existencia de factores bio-psicosociales también muy diversos y complejos, lo que obviamente tendrá como consecuencia también una heterogeneidad considerable en cuanto a los patrones de apego que puedan encontrarse, intentamos no obstante encontrar si hay algún patrón que pueda asociarse a la predisposición en general a desarrollar una dependencia de sustancias, del mismo modo que a nivel bioquímico se ha vinculado a neurotransmisores como la dopamina, los opioides endógenos o los endocannabinoides en la patogenia de muy diversos TCD.

Hemos buscado revisar las investigaciones donde se considera el apego como variable individual del sujeto consumidor de drogas, motivo por el cual no se han incluido en esta revisión los trabajos que entienden "apego" como "calidad de las relaciones", si bien la mayoría de ellos coinciden en señalar la relación entre el abuso de drogas en los adolescentes, y la existencia de una escasa cohesión familiar y un estilo de crianza basado en el "control sin afectividad"1-2.

En general, los estudios que revisaremos tratan de evaluar tanto "representaciones de apego", mediante entrevistas, como "estilos de apego", usando para ello instrumentos autoaplicados, si bien ambos tipos de valoración extraen datos que sólo son moderadamente comparables entre sí $^{3}$. Estas pautas de apego se definen como "patrones sistemáticos de expectativas, necesidades, emociones, estrategias de regulación de las emociones, y conducta social que resulta de la interacción de un sistema conductual de apego innato y una historia particular de experiencias de apego, generalmente empezando en las relaciones con los padres" 3 . Dada la escasez de trabajos que aborden el tema del apego, hemos incluido (a excepción de la salvedad especificada anteriomente) todos los trabajos que hemos encontrado sobre el tema del apego en sujetos con TCDs, tanto en búsqueda en PubMed como en Google, así como los artículos citados en referencias de los encontrados anteriormente, que servían en nuestro objetivo.

Nuestra hipótesis de trabajo es la existencia en general de apegos no seguros entre los sujetos con TCDs, de modo que mantendrían su consumo bien como una forma de romper con el entorno (estrategia devaluadora) como de evitar relaciones personales maduras a la vez que se mantienen en una necesidad constante de cuidados (estrategia temerosa o evasiva)

\section{INSTRUMENTOS PARA LA VALORACIÓN DEL APEGO}

Cada una de las tres líneas de trabajo creadas para valorar los estilos y representaciones de apego ha generado su propio instrumento. En la valoración del apego en consumidores de drogas se han usado los tres instrumentos, que son: el cuestionario o instrumento autoaplicado de Hazan y Shaver ${ }^{4}$ (Hazan and Shaver self-report measure, HSSR); el modelo de Bartholomew5; y la Entrevista de apego del adulto (Adult Attachment Interview, AAl) de Main y Goldwin 6 .

\section{Cuestionario autoaplicado de Hazan y Shaver (HSSR)}

Hazan y Shaver ${ }^{4}$ diseñaron un instrumento autoaplicado que consiste en descripciones breves de 3 estilos de apego, con respecto a las experiencias en las relaciones amorosas. Los sujetos tienen que puntuar primero con cuál de las 3 descripciones de estilos de apego se sienten más identificados (puntuando sólo 
una) y posteriormente puntuar en una escala likert de 1 a 7 el grado de desacuerdo o acuerdo (respectivamente) con cada uno de los estilos. Los estilos de apego se llamaron seguro, ansioso-ambivalente y evasivo, en correspondencia con la clasificación original de Ainsworth del apego en niños ${ }^{7}$.

Hasta el momento el único estudio que usa el HSSR con una muestra clínica de adictos a drogas es el de Finzi-Dottan y cols. ${ }^{8}$. Usaron la versión hebrea del HSSR en una muestra de hombres israelíes casados. La media de edad de los 56 participantes era 39 años y tenían una larga historia de consumo y dependencia de drogas. Aproximadamente la mitad habían consumido heroína, una cuarta parte heroína y cocaína, y el resto otras combinaciones de drogas. El estudio se llevó a cabo tras la desintoxicación. La mayoría (61\%) de los participantes se clasificaron como evasivos, $27 \%$ como seguros y sólo $12 \%$ como ansiosos-ambivalentes. Algunos autores han dudado sobre la posibilidad de usar instrumentos autoaplicados en este tipo de "muestras difíciles de estudiar"9-10 pero los resultados son coherentes con los vistos en muestras no clínicas (ver más abajo) con el mismo instrumento.

Cooper, Shaver y Collins ${ }^{11}$, usando el HSSR, estudiaron la relación entre los estilos de apego, la regulación de emociones, y diferentes tipos de problemas de conducta, en una muestra comunitaria representativa grande $(n=1989)$ de adolescentes de 13-19 años. Se evaluó el consumo de drogas con la prevalencia en 6 meses mediante autoinformes. Se vio la necesidad de diferenciar entre consumo de drogas experimental, o consumos más graves. El consumo experimental, que los autores interpretan como una conducta de exploración, mostró una tasa mayor entre sujetos seguros que entre los evasivos. En cambio, los consumos más graves se ven como parte de un patrón más amplio de problemas de conducta, que a su vez se valoran como un intento de enfrentarse al malestar.

Mickelson, Kessler y Shaver ${ }^{12}$ también usaron el HSSR, en una muestra amplia $(n=8098)$ representativa de Estados Unidos de adolescentes y adultos (15-54 años). La prevalencia-vida de los trastornos psiquiátricos, incluidos los TCD, se evaluaron como diagnósticos DSM-III-R basados en la entrevista estandarizada. Los trastornos psiquiátricos se asociaron en general al apego ansioso y evasivo. Los TCD fueron el único tipo de trastornos cuya relación era mayor con el apego evasivo que con el ansioso. Este estudio es importante por tratarse de una muestra amplia y representativa, por centrarse en TCD clínicamente relevantes y por el uso de entrevista estandarizada. Una limitación es que los TCD se evaluaron como prevalencia-vida, de modo que los trastornos posiblemente habían sucedido en décadas pasadas, mientras que el apego se valoraba como una categoría en el momento actual. Esto pudiera debilitar la relación entre apego y TCD, que pudiera ser incluso mayor en una muestra de consumidores actuales de drogas.

Uno de los primeros estudios con el HSSR ${ }^{13}$ examinó los estilos de apego y la relación conyugal entre parejas jóvenes recién casadas que participaron en un estudio longitudinal de consumo de alcohol y funcionamiento de la pareja ( $n=644)$. De modo similar a los hallazgos de otros estudios, los hombres evasivos era más probable que fueran bebedores importantes. En cambio, en las mujeres, el consumo de alcohol y el apego no estaban relacionados, si bien esto pudiera deberse a la baja frecuencia de consumo de alcohol femenino en la muestra.

Brennan y Shaver ${ }^{14}$ estudiaron el apego (valorado mediante el HSSR), la regulación del afecto y el funcionamiento en relaciones amorosas en una muestra de 178 universitarios. Se recogió mediante autoinformes la cantidad y la frecuencia de alcohol consumido así como los motivos para beber. Como era de esperar, los autores encontraron que el consumo social no se relacionaba con el apego. Sin embargo, "beber para enfrentarse a algo" se correlacionó inversamente con el apego seguro y directamente con el apego evasivo o ansioso. Además, los autores señalaron diferentes motivos de enfrentamiento en los dos grupos inseguros: los evasivos bebían para reducir la tensión y evitar la dependencia emocional, mientras que los ansiosos bebían para reducir la ansiedad. Y en general, consistente con otros estudios, el consumo más importante se relacionaba con el apego evasivo.

Por tanto, aunque los hallazgos de todos los trabajos que usaron el HSSR no son completamente consistentes, en la mayoría de ellos los consumos más graves se relacionaban con apego evasivo. De haber un sesgo en estos resultados, se debería a la evaluación autoinformada del consumo de drogas. Puesto que suele haber una tendencia de los sujetos evasivos a señalar menos conductas problemáticas y en cambio los ansiosos a señalar más ${ }^{15}$, esto conduciría a subestimar la relación entre el apego evasivo y el consumo de drogas, y a sobreestimar la relación entre apego ansioso y consumo, por lo que posiblemente la relación entre apego evasivo y consumo de drogas sea aún mayor y más clara de lo aparecido en estos estudios. Y si bien Mickelson y cols. ${ }^{12}$ redujeron este posible sesgo usando la entrevista estructurada SCID, para lograr una evaluación más válida de los TCD, es muy posible que las tendencias a subestimar pudieran también sesgar una entrevista clínica

\section{Entrevista del apego del adulto}

La Entrevista de apego del adulto (Adult Attachment Interview, AAI $)^{6,16}$ es una larga entrevista semiestructurada diseñada para clarificar recuerdos de la interacción en la infancia con los padres y evaluar las 
representaciones de apego. Es codificada en términos de la coherencia del discurso que muestra la persona mientras relata experiencias relevantes de su infancia, como también la estructura de su relato y su habilidad para colaborar efectivamente con el entrevistador. Requiere que el entrevistador se haya formado en un centro acreditado para impartir cursos sobre dicho instrumento (por ejemplo, el Centro Anna Freud de Hampstead, en Londres). La entrevista clasifica los estilos de apego basándose en la organización mental actual del sujeto, en cuanto a coherencia de pensamiento y sentimientos en cuanto al apego. Las cuatro categorías en AAl (seguro-autónomo, preocupado, devaluador y no resuelto) se diseñaron en paralelo a las clasificaciones de apego infantil (seguro, ambivalente, evasivo y desorganizado) de Bartholomew ${ }^{17}$, por la similitud de modelos de trabajo internos y estrategias defensivas empleadas ${ }^{16}$.

Rosenstein y Horowitz ${ }^{18}$ estudiaron una muestra de 60 adolescentes de 13-19 años, ingresados para tratamiento psiquiátrico, de los cuales 29 eran consumidores de drogas. Todos los participantes tenían diagnósticos principales diferentes del TCD. Se clasificaron en parte como devaluadores y en parte preocupados. No hubo asociación entre consumo de drogas y apego no resuelto. Los consumidores de drogas con trastornos de conducta (TC) tenían apegos devaluadores, mientras que los consumidores con trastornos afectivos eran clasificados como devaluadores o preocupados. Este estudio muestra la importancia de los trastornos psiquiátricos comórbidos, puesto que asume diferentes clasificaciones de apego, diferentes estados emocionales y diferentes patrones de consumo de drogas en adolescentes con TC frente a trastornos afectivos.

Allen, Hauser y Borman-Spurell19 estudiaron una muestra similar, de 66 pacientes psiquiátricos adolescentes ingresados con diagnósticos principales diferentes al TCD. Señalan una relación entre "consumo de drogas duras" y apego devaluador, sin encontrar asociación con los apegos preocupado o no resuelto. Sin embargo, no hay información sobre cuántos de esos 66 casos eran de consumidores de drogas, sobre tipo de drogas consumidas ni cómo se evaluó.

Fonagy y cols. ${ }^{20}$ estudiaron una muestra de 82 pacientes psiquiátricos adultos no psicóticos, la mayoría mujeres (82\%). De ellos un 37\% tenía también TCD. Usando una versión de 3 categorías del AAI, los consumidores de drogas se clasificaron fundamentalmente como preocupados. Cuando se incluyó la categoría adicional de "trauma no resuelto", la mayoría de consumidores de drogas estaban dentro de ella. No obstante, los autores no informan del tipo de drogas, patrones de consumo de drogas o los diagnósticos principales de los consumidores de drogas.
Riggs y Jacobvitz ${ }^{21}$ entrevistaron con el AAl una muestra no clínica de 233 parejas, cuya media de edad era de 30 años, que esperaban un hijo; de ellas, 26 eran consumidores de drogas. La prevalencia vida de TCD se evaluó con diagnósticos DDSM-III-R. En la versión de 3 categorías no había relación entre TCD y clasificaciones de apego. En la de 4 categorías, los TCD se relacionaban de nuevo con traumas no resueltos.

Los estudios con AAl indican que existiría un apego inseguro en los consumidores de drogas, pero sin una categoría única que se relacione siempre con los TCD. La mayoría de estudios evaluaron TCD como diagnósticos DSM-III-R para minimizar el sesgo debido a declarar menores consumos de droga. Sin embargo, hay una serie de limitaciones metodológicas que dificultan o incluso podrían ser las responsables de los problemas para extraer conclusiones nítidas. Por ejemplo, ninguno de estos estudios con AAl se diseñó para informar sobre TCD en particular (todos los participantes en los tres estudios clínicos tenían un diagnóstico principal, aparte del TCD), y el número de participantes con TCD era pequeño o incluso no se especificaba. Las relaciones entre estos trastornos primarios, TCD y clasificaciones de apego no se diferenciaban por lo general. Tampoco ninguno de los estudios daba información sobre la gravedad del TCD o las drogas consumidas. $Y$, finalmente, las muestras eran diferentes en edad, distribución por géneros, y diagnósticos

\section{Entrevista e instrumentos autoaplicados de Bartholomew}

Bartholomew ${ }^{5,17}$ desarrolló un modelo de cuatro categorías de apego, basado en modelos de trabajo internos positivos y negativos del yo y los demás. Bartholomew adoptó los patrones seguro y preocupadoansioso de anteriores abordajes, pero diferenció entre dos categorías de evasivo: evasivo temeroso (similar al evasivo del HSSR) y evasivo devaluador o rechazante (según el AAI). Bartholomew desarrolló varios instrumentos autoaplicados (para evaluar los estilos de apego definidos arriba), donde se trataba de indicar con cuál de los 4 estilos de apego se sentía más identificado el sujeto y posteriormente en qué grado (en escala likert de 7 puntos) se mostraba de acuerdo o no con cada uno de los estilos; y entrevistas de apego (para valorar las representaciones del apego, en la infancia y en las actuales relaciones de apego).

McNally y cols. ${ }^{22}$ estudiaron el apego, el consumo de alcohol y los problemas que afrontar que motivaban el consumo, en una muestra de 366 universitarios, usando unos índices dimensionales del modelo de trabajo interno del yo y los demás, basados a su vez en el instrumento autoaplicado de Bartholomew. El nivel de consumo de alcohol en el mes previo, los problemas relacionados con la bebida, y las cuestiones que afrontar que motivaban el consumo, se reco- 
gieron mediante autorregistros. Un modelo del yo (pero no modelo de los demás) negativo, se encontró que se relacionaba con problemas relacionados con la bebida, consecuencias negativas y "beber para afrontar problemas". En términos de estilo de apego, esto significa que los apego temeroso y preocupado se relacionaban con un uso más problemático del alcohol, que no estaba presente en sujetos con apego seguro ni devaluador.

Magai ${ }^{23}$ estudió el consumo de alcohol y el apego con la entrevista de Bartholomew. Entre otras cosas, el estudio evaluó la frecuencia del consumo de alcohol y los motivos para dicho consumo, con autorregistros. Los diferentes motivos para beber se asociaron también con diferentes categorías de apego. Los sujetos preocupados bebían para reducir estados de ánimo negativos, mientras los devaluadores bebían para mejorar estados de ánimo positivos. La frecuencia de consumo se relacionó directamente con el apego preocupado.

El trabajo más reciente con la entrevista de Bartholomew ${ }^{24}$ incluyó una muestra de 71 adolescentes dependientes de opiáceos, de 14-25 años, a los que comparó con 39 controles de población no clínica. El apego se valoró mediante entrevista, y el consumo de drogas se valoró con una escala de gravedad y con determinación de tóxicos en orina, que eliminaría los sesgos derivados de los autoinformes de consumo. En los adolescentes drogodependientes (ADD) el apego predominante fue el temeroso, mientras que los controles fueron predominantemente seguros; las diferencias entre ambos grupos en apego temeroso y seguro fueron amplias y significativas. Sin embargo, no hubo diferencias en cuanto a apego preocupado o devaluador. En cuanto a la gravedad de la adicción en ADD, se correlacionó directamente con el apego temeroso, pero inversamente con apego devaluador. Estos resultados apoyan la validez de la distinción de Bartholomew ${ }^{17}$ entre evasivo devaluador y evasivo temeroso. Se vieron también diferencias significativas en el apego entre ADD y controles, incluso controlando por edad, como si un apego temeroso sea un factor de riesgo para dependencia de drogas que a su vez altere el desarrollo normal del apego seguro conforme avanza la adolescencia. De hecho, los hermanos de ADD en este trabajo eran menos temerosos y más seguros que los ADD, si bien no hubo diferencias en apego preocupado o devaluador, y de todos modos hay que recordar que los hermanos que participaron representaron una selección de todos los hermanos. En cuanto a la presencia de trastornos comórbidos, no se asoció con la gravedad de la adicción, pero sí hubo relación entre el apego temeroso y la gravedad de la adicción cuando había además trastornos comórbidos, lo cual sugiere que el apego temeroso podría no ser específico de consumidores de drogas pero sí asociarse de modo más general con problemas psiquiátricos.
Como vemos, hay poca investigación con el modelo de Bartholomew. Los primeros estudios con autorregistros de Bartholomew, con consumidores de alcohol ${ }^{22-23}$ indicaban relación entre mayor consumo de alcohol y estilos de apego preocupados y temerosos, pero dado que el consumo de alcohol se recogía con autorregistros, los resultados de nuevo pueden estar sesgados por haber reconocido consumos mayores los sujetos con apegos preocupados. El último estudio, que señala apego evasivo temeroso en ADD de heroína, puede considerarse consistente parcialmente con ambos estudios, pero también con los trabajos realizados con el HSSR, dado que el apego evasivo del HSSR se asume que corresponde con el evasivo temeroso de Bartholomew.

\section{ESTUDIOS SOBRE APEGO Y TCD EN LA ADOLES- CENCIA}

Resumiendo los resultados de los estudios existentes, los TCD se asocian de modo consistente con apego inseguro pero los resultados sobre cuáles categorías específicas de apego inseguro, son inconsistentes según los instrumentos. Los estudios con HSSR indican una relación relativamente consistente entre apego evasivo y consumo de drogas. Vistos globalmente, los estudios con AAl produjeron resultados inconsistentes, donde el consumo de drogas se relacionó con apego preocupado ${ }^{18,20}$ y devaluador ${ }^{18-19}$, si bien dos estudios con AAl ${ }^{20-21}$ indican una relación entre apego no resuelto y subgrupos de consumidores adultos de drogas. Los pocos estudios con el modelo de Bartholomew indican una relación cuestionable entre mayor consumo de alcohol y apego preocupado y temeroso en muestras no clínicas.

Todos los estudios revisados son transversales, por lo que no hay información sobre la dirección de la relación entre apego inseguro y TCD. Parece plausible que el apego inseguro, en general desarrollado en la infancia, es un factor de riesgo de TCD que en general se desarrolla en la adolescencia. El apego inseguro se ha revelado factor de riesgo de diversos problemas de salud mental ${ }^{25}$. Pero también los TCD tienen un fuerte impacto negativo en la calidad de las relaciones $y$, especialmente en muestras de consumidores de drogas de larga evolución ${ }^{8}$ el apego inseguro podría ser en parte consecuencia del consumo de drogas.

La mayoría de estudios se basan en autoinformes del consumo de drogas. Dado que el consumo de drogas generalmente no es una conducta socialmente aceptada, y que el uso de drogas ilegales es incluso un delito, los autoinformes pueden conducir a una subestimación de la gravedad del consumo. Las entrevistas clínicas son más fiables, ya que el entrevistador estima la gravedad del consumo de drogas pero depende 
también de lo que el participante diga al entrevistador. Este punto es especialmente importante con respecto al apego debido a las tendencias a reconocer menores consumos por parte de los individuos devaluadores (ver arriba) ${ }^{15}$, lo cual podría evitarse con la determinación de tóxicos de orina para valorar los consumos ${ }^{24}$.

Aunque las diferentes drogas crean diferentes estados emocionales, no sabemos si podrían relacionarse diferentes categorías de apego al uso de drogas diferentes. Con una amplia variedad de drogas psicoactivas disponibles, parece probable que la gente elige una o más drogas según su efecto emocional. De momento no hay comparaciones sistemáticas entre los consumidores de drogas diferentes en cuanto al apego.

Las cosas se complican más aún, porque hay diferentes motivos principales para consumir drogas con diferentes niveles de gravedad. Según Moffitt ${ }^{26}$, en el desarrollo normal de la adolescencia, se produce un consumo experimental de drogas, a menudo incluyendo episodios de consumo importante y/o conductas problemáticas de otro tipo, y típicamente en un contexto con otros adolescentes. Este consumo de drogas "limitado a la adolescencia" viene motivado principalmente por intentos de enfrentarse a una situación de malestar emocional ${ }^{27}$ y no implica antecedentes o consecuencias patológicas ${ }^{26}$. Este modelo es consistente con el hallazgo de una relación entre apego seguro y consumo de drogas experimental en la adolescencia que se considera una conducta de exploración ${ }^{11}$. Sin embargo, aproximadamente $10 \%$ de los adolescentes consumidores muestran patrones más graves de consumo y dependencia que persisten en la edad adulta ${ }^{26}$. ¿Cuál sería su estilo de apego? La simple observación de la conducta actual del paciente podría llevarnos a asumir a priori la existencia de estilos de apego inseguros, por ejemplo evitativos, ya que cuando continúa el consumo de drogas más allá de la adolescencia, entran en acción los procesos adictivos mismos (craving, miedo del abandono, dependencia psicológica y fisiológica) que se hacen cada vez más importantes y podrían crearnos confusión sobre las razones originales del consumo de drogas ${ }^{28}$, por lo que desde la teoría del apego, sería más fructífero estudiar las fases iniciales del TCD, cuando el consumo de droga tiene ya relevancia clínica pero su motivación no está aún influida por procesos adictivos, y de no ser posible (en la mayoría de casos), utilizar instrumentos estandarizados como los que hemos visto anteriormente.

Los adolescentes con TCD tienen tasas altas de comorbilidad con otros problemas de salud mental, como trastornos depresivos y ansiosos, y trastornos de conducta ${ }^{27}$. La combinación de TCD y trastorno de conducta está relacionada con impulsividad, agresividad, problemática social, fracaso escolar, conflictos familiares y ruptura familiar. Rosenstein y Horowitz ${ }^{18}$ señalan principalmente clasificaciones devaluadoras en este grupo. En cuanto a los TCD en sujetos con trastornos depresivos y ansiosos, podrían considerarse intentos de enfrentarse con los estados emocionales típicos de estos trastornos; de nuevo Rosenstein y Horowitz ${ }^{18}$ son los únicos que proporcionan datos sobre el apego en este grupo, señalando que los adolescentes con TCD y trastornos afectivos se clasifican en parte como devaluadores y en parte como preocupados, pero no explican este resultado. Por tanto necesitamos más estudios para comprender las complejas interrelaciones entre apego, TCD y trastornos comórbidos.

En la literatura hay descrita una alta proporción de consumidores de drogas que han experimentado abuso sexual o maltrato físico en la infancia ${ }^{29}$ y de nuevo el consumo de drogas se puede considerar un intento de enfrentarse al malestar emocional causado por estas experiencias. En esta línea, dos estudios encontraron relación entre TCD y apego no resuelto en muestras de adultos ${ }^{20-21}$, mientras que otros dos estudios no encuentran esta relación en muestras adolescentes $^{18-19}$. Estos resultados apuntan que la edad podría ser una variable importante, aunque no está claro por qué habría más sujetos adultos que adolescentes con traumas no resueltos. Quizá el propio estado mental alterado asociado al consumo de drogas puede dificultar la elaboración de dichos traumas ${ }^{21}$ pero esta hipótesis debería estudiarse específicamente.

\section{APEGO Y TCD: DISCUSIÓN Y CONCLUSIONES}

Ahora intentaremos integrar los resultados sobre las diversas categorías de apego, recordando que la correlación entre las clasificaciones de apego en el AAl, la entrevista de Bartholomew, y los diferentes instrumentos autoaplicados es sólo pequeña ${ }^{3}$. Las entrevistas de apego evalúan las representaciones del apego y se centran en las reglas para el procesamiento de información relacionada con el apego y en los procesos defensivos. Los instrumentos autoaplicados evalúan estilos de apego y se centran en la conducta interpersonal en las relaciones amorosas.

El apego seguro es más o menos el mismo en todos los instrumentos y no parece jugar ningún papel para explicar los TCD. La categoría de apego correspondiente a la categoría original de apego infantil ansioso/ambivalente de Ainsworth y cols. ${ }^{7}$ se denomina igual en el HSSR, pero se llama preocupado en el modelo de Bartholomew y en el AAl. La categoría de apego que corresponde con la evasiva de la clasificación original de Ainsworth se llama devaluadora en el AAl y en el modelo de Bartholomew. El apego evasivo en el HSSR se define diferente, y corresponde a la categoría evasiva-temerosa de Bartholomew. Shaver y Mikulincer ${ }^{3}$ discuten en detalle los diferentes conceptos de apego evasivo, temeroso y devaluador. 
Hay pocos datos empíricos que apoyen una relación entre el apego preocupado o ansioso y los TCD. Los sujetos que se consideran preocupados en el AAl muestran una preocupación exagerada, confusa e irritada con las experiencias y figuras de apego y en reacción a la inseguridad de apego tienden a usar estrategias de hiperactivación del sistema de apego ${ }^{30}$ buscando la cercanía en las relaciones de apego ${ }^{4}$. No creemos que los sujetos con TCD usen ninguna de estas estrategias. El consumo de droga cuando constituye una conducta persistente y no simplemente exploratoria, parece más bien formar parte de una estrategia desactivadora y distanciadora de las relaciones de apego. Los estudios más recientes cuestionan la importancia del impacto de los amigos ${ }^{27}$ puesto que las relaciones con otros ADD del entorno de consumo, mal pueden considerarse como búsqueda de cercanía en el sentido de la teoría del apego, pues no parecen ser lo bastante seguras, estables y duraderas. Cuando consideramos las relaciones familiares, esto es aún más claro: el consumo de drogas en la adolescencia se ha visto que se asocia con abandono de las relaciones y marginación social, con escasos lazos con la familia y conflictos familiares y rupturas ${ }^{31}$.

Las pruebas empíricas de una relación entre apego devaluador y TCD sólo aparecen en dos estudios con AAl18-19. Además podría haber un número desconocido de sujetos devaluadores en la categoría evasiva del HSSR. No hay pruebas de que haya relación entre apego evasivo y TCD en los estudios que usan el modelo de Bartholomew. La relación entre apego devaluador y TCD pudiera estar subestimada por menor reconocimiento del consumo de drogas (ver arriba). Los sujetos que se clasifican como devaluadores en el AAl dicen haber tenido experiencias positivas con los cuidadores, a menudo los idealizan pero no pueden apoyar este alegato e insisten en la falta de recuerdos de la infancia. Activamente devalúan las necesidades, experiencias y relaciones de apego ${ }^{16}$. Los conceptos de estilo de apego describen que confían en sí mismos y son distantes en las relaciones interpersonales ${ }^{5}$. Este tipo de conducta interpersonal es coherente con los hallazgos de abandono, marginación social y escasos lazos con la familia, de los consumidores de drogas ${ }^{31}$. Hay una diferencia importante entre lo que sabemos sobre TCD y apego devaluador. Los sujetos devaluadores se enfrentan con el malestar relacionado con el apego desactivando su sistema de apego $^{30}$. La diferencia esencial de los ADD con apego devaluador es que los sujetos con TCD parecen incapaces de desactivar su sistema de apego sin recurrir al uso de drogas.

La mayoría de los estudios que usan el HSSR suelen indicar una relación entre TCD y apego evasivo. El AAl no incluye una categoría aparte para este apego evasivo, que corresponde al evasivo temeroso del modelo de Bartholomew. Shaver y Mikulincer ${ }^{3}$ seña- lan las diferencias entre apego devaluador y temeroso: "mientras que en el evasivo devaluador funcionan adecuadamente las estrategias de desactivación e inhibición del reconocimiento de las señales relacionadas con peligro y las necesidades de apego, en el evasivo temeroso pueden fracasar estas estrategias en condiciones muy estresantes. (En tales situaciones) los sujetos evasivos temerosos desean la cercanía a figuras de apego, aunque se sienten incapaces de confiar y apoyarse en ellas. Esto puede hacer que sus sistemas de apego sigan activados mientras que sus estrategias conductuales sugieren desactivación". En cambio, en la vida diaria, no ven (como sí hacen los preocupados) "la búsqueda de cercanía como una opción viable"3 y no adoptan una estrategia de hiperactivación. También, al contrario que los sujetos devaluadores, no tienen mecanismos de defensa de tipo desactivador para enfrentarse al malestar emocional relacionado con el apego. De tal modo que, desde el punto de vista del apego, el consumo de drogas puede verse como un intento de enfrentarse con la inseguridad del apego, para disminuir el malestar emocional y regular las relaciones interpersonales. Por tanto, en teoría, la categoría de apego que mejor se ajusta con TCD es el evasivo temeroso. $Y$ dado que empíricamente, la relación mejor establecida es entre el apego evasivo en el HSSR y los TCD; y que la definición de estas dos categorías de apego es muy parecida, parece probable una asociación entre el evasivo temeroso y los TCD.

En general la presencia de estos tipos de apego inseguros, o de un apego evasivo temeroso creemos que podría tiene relevancia clínica en cuanto a la relación terapéutica, pudiendo explicar, desde el punto de vista del apego, buena parte de las dificultades para establecer y mantener una alianza terapéutica con ADD, que por otro lado sería el factor crucial en el tratamiento de los drogodependientes. En el futuro sería interesante estudiar la relación entre apego y pronóstico del tratamiento.

Los hallazgos encontrados han de ser tomados con precaución, ya que se trata de estudios muy heterogéneos, que han usado instrumentos diferentes para la valoración del apego, y en general las muestras de sujetos estudiados son también muy diferentes en cuanto a edad, tipo de sustancia consumida, etc. y por último, muchos de estos estudios omiten la gravedad del consumo, o incluso no especifican el tipo de sustancias consumidas.

Como conclusiones de la revisión, encontramos por tanto una presencia de representaciones y estilos de apego inseguros en los sujetos con TCDs. Si bien algunos estudios apuntan a la existencia de patrones devaluadores de apego, la mayoría de trabajos encuentran apegos predominantemente evasivos o temerosos. 


\section{REFERENCIAS}

1. Lee JM, Bell NJ. Individual differences in attachmentautonomy confitgurarion. Linkages with substance use and youth competencies. J Adolesc 2003; 26: 347-61.

2. McArdle P, Wiegersma A, Gilvarry E, Kolte B, McCarthy $S$, Fitzgerald $\mathrm{M}$ et al. European adolescent substance use: the roles of family structure, functin and gender. Addiction 2002; 97: 329-36.

3. Shaver PR, Mikulincer M. Attachment-related psychodinamics. Attachm Hum Dev 2002; 4: 133-61.

4. Hazan C, Shaver P. Conceptualizing romantic love as an attachment process. J Pers Soc Psychol 1987; 52: 511-24.

5. Bartholomew K, Horowitz LM. Attachment styles among young adults: a test of a four-category model. J Pers Soc Psychol 1991; 61: 226-44.

6. Hesse E. The adult attachment interview: Historical and current perspectives. In J. Cassidy \& PR Shaver (eds.), Handbook of attachment. New York: Guilford, 1999, pp.336-54.

7. Ainsworth MD, Blehar MC, Waters E, Wall S. Patterns of attachment: assessed in the strange situation and at home. Hillsdale, NJ: Erlbaum, 1978.

8. Finzi-Dottan R, Cohen O, Iwaniec D, Sapir Y, Weizman A. The drug-user husband and his wife: attachment styles, family cohesión and adaptability. Subst Use Misuse 2003; 38 (2): 271-92.

9. Bartholomew K, Moretti M. The dynamics of measuring attachment. Attachm Hum Dev 2002; 4: 162-5.

10. Bifulco A. Attachment style measurement: a clinical and epidemiological perspective. Attachm Hum Dev 2002; 4: $180-88$.

11. Cooper ML, Shaver PR, Collins NL. Attachment styles, emotion regulation and adjustment in adolescence. $J$ Pers Soc Psychol 1998; 74: 1380-97.

12. Mickelson KD, Kessler RC, Shaver PR. Adult attachment in a nationally representative sample. J Pers Soc Psychol 1997; 73: 1092-1106.

13. Senchak M, Leonard KE. Attachment styles and marital adjustment among newlywed copules. J Soc Pers Relat 1992; 9: 61-4.

14. Brennan KA, Shaver PR. Dimensions of adult attachment, affect regulation and relationship functioning. Pers Soc Psychol Bull 1995; 21: 267-83.

15. Crowell JA, Fraley RC, Shaver PR. Measurement of individual differences in adolescent and adult attachment. In J. Cassidy \& PR Shaver (eds.), Handbook of attachment. New York: Guilford, 1999.

16. Main M, Goldwyn R. Adult attachment scoring and classification system. Manuscrito no publicado, Universidad de Berkeley (California), 1998.

17. Bartholomew K. Avoidance of intimacy. An attachment perspective. J Soc Pers Relat 1990; 7: 147-78.

18. Rosenstein SD, Horowitz HA. Adolescent attachment and psychopathology. J Cons Clin Psychol 1996; 64 (2): 244-53.
19. Allen JP, Hauser ST, Borman-Spurell E. Attachment theory as a framework for undesrstanding sequelae of severe adolescent psychopathology: an 11-year followup study. J Cons Clin Psychol 1996; 64: 254-63.

20. Fonagy P, Leigh T,Steele M, Steele H, Kennedy R, Mattoon $G$ et al. The relation of attachment status, psychiatric classification and response to psychotherapy. J Cons Clin Psychol 1996; 64: 22-31.

21. Riggs SA, Jacobvitz D. Expectant parents' representations of early attachment relationships: associations with mental health and family history. J Cons Clin Psychol 2002; 70: 195-204.

22. McNally AM, Palfai TP, Levine RV, Moore BM. Attachment dimensions and drinking-related problems among adults: the mediational role of coping motives. Addict Behav 2003; 28 (6): 115-27.

23. Magai C. Affect, imagery and attachment: working models of interpersonal affect and the socialization of emotion. In J. Cassidy \& PR Shaver (eds.), Handbook of attachment. New York: Guilford, 1999; pp. 787-802.

24. Schindler A, Thomasius R, Sack PM, Gemeinhardt B, Kustner U, Eckert J. Attachment and substance use disorders: a review of the literature and a study in drug dependent adolescents. Attach Hum Dev 2005; 7 (3): 207-28.

25. Dozier M, Stovall KC, Albus KE. Attachment and psychopathology in adulthood. In J. Cassidy \& PR Shaver (eds.), Handbook of attachment. New York: Guilford, 1999; pp. 497-519.

26. Moffit TE. Adolescence-limited and life-course-persistent antisocial behavior: a developmental taxonomy. Psychol Rev 1993; 100: 674-701.

27. Weinberg NZ, Rahdert E, Colliver JD, Glantz MD. Adolescent substance abuse: a review of the past 10 years. J Am Acad Child Adolesc Psychiatry 1998; 37 (3): 252-61.

28. Shaffer HJ, Robbins M. Psychotherapy for addictive behavior: a stage-change approach. In AM Washton (ed.), Psychotherapy and substance abuse: A practitioner's handbook. New York: Guilford, 1995; pp.103-123.

29. Clark DB, Lesnick L, Hegedus AM. Traumas and other adverse life events in adolescents with alcohol abuse and dependence. J Am Acad Child Adolesc Psychiatry 1997; 36: 1744-51.

30. Kobak RR, Cole HE, Ferenz-Gillies R, Fleming WS, Gamble $W$. Attachment and emotion regulation during motherteen problem-solving. A control theory analysis. Child Dev 1993; 64: 231-45.

31. Hawkins JD, Catalano RF, Miller JY. Risk and protective factors for alcohol and other drug problems in adolescence and early adulthood: implications for substance abuse prevention. Psychol Bull 1992; 112 (1): 64-105. 


\section{APÉNDICE. ESTUDIOS SOBRE APEGO Y CONSUMO DE DROGAS}

Tabla 1. Estudios con el instrumento autoaplicado de Hazan y Shaver.

\begin{tabular}{|c|c|c|c|c|}
\hline Autores y año & Muestra, edad & $\begin{array}{c}\text { N consumidores del } \\
\text { total }\end{array}$ & Droga, gravedad & $\begin{array}{l}\text { Estilo de apego } \\
\text { ligado al consumo }\end{array}$ \\
\hline $\begin{array}{l}\text { Finzi-Dottan y cols. } \\
2003^{8}\end{array}$ & $\begin{array}{c}\text { Clínica, toxicómanos } \\
\text { desintoxicados } \\
39 \text { años }\end{array}$ & 56 de 56 & $\begin{array}{l}\text { Heroína y otros, } \\
\text { adicción larga }\end{array}$ & Evasivo \\
\hline Cooper y cols. $1998^{11}$ & $\begin{array}{l}\text { Comunitaria } \\
\text { representativa, } \\
\text { 13-19 años }\end{array}$ & 1151 de 2011 & $\begin{array}{l}\text { Alcohol, drogas. } \\
\text { Experimental y } \\
\text { patológico }\end{array}$ & $\begin{array}{c}\text { Experimental: seguro, } \\
\text { ansioso } \\
\text { Patológico: ansioso } \\
\text { (evasivo) }\end{array}$ \\
\hline $\begin{array}{c}\text { Mickelson y cols. } \\
1997^{12}\end{array}$ & $\begin{array}{c}\text { Representativa de } \\
\text { EEUU, } \\
15-24 \text { años }\end{array}$ & 2876 de 8098 & $\begin{array}{c}\text { Alcohol, drogas. } \\
\text { Abuso y dependencia }\end{array}$ & Evasivo temeroso \\
\hline $\begin{array}{c}\text { Senchak y Leonard } \\
1992^{13}\end{array}$ & $\begin{array}{l}\text { No clínica: parejas } \\
\text { recién casadas, } \\
24 \text { años }\end{array}$ & ¿? de 644 & Alcohol, ¿? & $\begin{array}{l}\text { Hombres evasivos, } \\
\text { mujeres sin relación }\end{array}$ \\
\hline $\begin{array}{c}\text { Brennan y Shaver } \\
1995^{14}\end{array}$ & $\begin{array}{l}\text { Universitarios, } \\
19 \text { años }\end{array}$ & 178 de 242 & Alcohol, ¿? & Evasivo \\
\hline
\end{tabular}

Tabla 2. Estudios con la Entrevista de apego del adulto (AAI)

\begin{tabular}{|c|c|c|c|c|}
\hline Autores y año & Muestra, edad & $\begin{array}{c}\text { N consumidores del } \\
\text { total }\end{array}$ & Droga, gravedad & $\begin{array}{c}\text { Estilo de apego } \\
\text { ligado al consumo }\end{array}$ \\
\hline $\begin{array}{c}\text { Rosenstein y } \\
\text { Horowitz, 1996 }\end{array}$ & $\begin{array}{c}\text { Psiquiátrica, 13-19 } \\
\text { años }\end{array}$ & 29 de 60 & $\begin{array}{c}\text { Devaluador o } \\
\text { preocupado }\end{array}$ \\
\hline Allen y cols. 1996 ${ }^{19}$ & $\begin{array}{c}\text { Psiquiátrica, 14-25 } \\
\text { años }\end{array}$ & $i ?$ de 66 & Drogas duras, $i ?$ & Devaluador \\
\hline Fonagy y cols. 199620 & $\begin{array}{c}\text { Psiquiátrica, adultos, } \\
82 \% \text { mujeres }\end{array}$ & 37 de 82 & $\begin{array}{c}\text { No resuelto, abuso } \\
\text { preocupado }\end{array}$ \\
\hline $\begin{array}{c}\text { Riggs y Jacobvitz, } \\
2002^{21}\end{array}$ & $\begin{array}{c}\text { No clínica, futuros } \\
\text { padres de clase } \\
\text { media, 30 años }\end{array}$ & 26 de 233 & Alcohol y drogas, \\
abuso & No resuelto \\
\hline
\end{tabular}

Tabla 3. Estudios con el modelo de Bartholomew.

\begin{tabular}{|c|c|c|c|c|}
\hline Autores y año & Muestra, edad & $\begin{array}{c}\mathbf{N} \text { consumidores del } \\
\text { total }\end{array}$ & Droga, gravedad & $\begin{array}{c}\text { Estilo de apego ligado } \\
\text { al consumo }\end{array}$ \\
\hline $\begin{array}{c}\text { McNally y cols. } \\
2003^{22}\end{array}$ & $\begin{array}{l}\text { Universitaria, } 18-23 \\
\text { años }\end{array}$ & 366 de 366 & Alcohol, consumo & Preocupado temeroso \\
\hline Magai, $1999^{23}$ & No clínica, ¿? & ¿? De ¿? & $\begin{array}{c}\text { Alcohol; leve, } \\
\text { moderado o grave }\end{array}$ & Preocupado o devaluador \\
\hline $\begin{array}{l}\text { Schindler y cols } \\
2005^{14}\end{array}$ & $\begin{array}{c}\text { Clínica terapia } \\
\text { familiar, } \\
14-25 \text { años }\end{array}$ & 71 de 17 & $\begin{array}{l}\text { Heroína y otras, } \\
\text { dependencia }\end{array}$ & Temeroso \\
\hline
\end{tabular}


